\title{
From Business Process Management to Flexible Image Analysis Applications: A Case Study
}

\author{
Faezeh Rohani ${ }^{1}$, Mohammad Amin Moghaddasi Far ${ }^{1}$, Fatemeh Fazayeli Bavojdan ${ }^{2}$ \\ ${ }^{1}$ Computer Science Department, Sanabad Golbahar Institute of Higher Education, Golbahar, Iran \\ ${ }^{2}$ Computer Science Department, Khayyam University, Mashhad, Iran
}

\section{Email address:}

faezeh.rohani@ut.ac.ir (F. Rohani), Amin.moghaddasi@ut.ac.ir (M. A. M. Far), ffazaelibavojdan@gmail.com (F. F. Bavojdan)

\section{To cite this article:}

Faezeh Rohani, Mohammad Amin Moghaddasi Far, Fatemeh Fazayeli Bavojdan. From Business Process Management to Flexible Image Analysis Applications: A Case Study. Computational Biology and Bioinformatics. Vol. 3, No. 3, 2015, pp. 40-44.

doi: $10.11648 /$ j.cbb.20150303.11

\begin{abstract}
The Business Process Management (BPM) as an advanced paradigm in workflow management has been very a very active research topic in the field software engineering and process management. The goal of this contribution is to take BPM advantages into the image processing -in particular medical image analysis- to provide a consistent and comprehensive software framework. A case study is also presented in this paper and the possibility for applying BPM on image analysis is specified separately. Furthermore, several quality attributes are addressed to create modifiable, reusable, and flexible framework for the medical imaging community. The present research is expected to draw attentions from medical image analysis to BPM, and make possible future enhancements in BPM's application.
\end{abstract}

Keywords: Business Process Management, Image Analysis, Software Engineering

\section{Introduction}

Image analysis as the intersection of signal processing, mathematical approaches, and machine perception has made a very big advance in many areas of application consisting biomedicine, medicine, industry, and entertainment by assisting the extraction of syntax and semantic information from digital images. The task of image analysis may go from low level image processing such as image sharpening and contrast enhancement, to medium and high level analysis including image segmentation, image registration, object detection and localization [1], [2].

In the other side, Business Process Management (BPM) has been a very well-known structure which could widely manage the entire processes of organizations. Business process management includes concepts, methods, and techniques to support the design, administration, configuration, enhancement, and analysis of business processes [3]. Business Process Management has received considerable attentions recently by both business administration and computer science communities. The basis of business process management is the explicit representation of business processes with their activities and the execution constraints between them. Once business processes are defined, they can be subject to process analysis, and its improvement. Information Technology and in particular Information Systems has made and a big advance in Business Process Management, and the reason is that more and more activities that a company performs have been supported by Information Systems. The goal of BPM is to reduce human errors and miscommunications, and focus stakeholders on the requirements of their roles.

While digital image analysis algorithms and its applications have been investigated for a long period, the use of BPM strategy in image analysis and in particular in image analysis software applications has been limited so far. In this work a study is performed to apply BPM to image analysis software applications as well as the image analysis work flow system. As a case study, we examine a highly-employed modular image analysis work flow system called Anima [4]. Anima has been designed for comprehensive and platform independent image analysis development. The present contribution is expected to draw attentions from BPM to digital image analysis community.

The major contributions of the paper are as follows:

1) The main contribution of the paper is to introduce BPM 
strategy based approach to the digital image analysis community. By applying BMP on image analysis we can increase several quality attributes of the image analysis workflows and software applications.

2) Investigating and examining Anima as a widely used image analysis workflow system regarding the BMP attributes is the next outcome of the work.

3) As the most important contribution, this paper provides several insights for incorporating BMP and digital image analysis.

The rest of the work is arranged as follows. In section 2, some recently related works in the area of digital image analysis as well as BMP are reviewed. The proposed case study, Anima, is described briefly in Section 3. We examine the Anima from different perspectives regarding the BMP based strategy and address several quality attributes as the desirable enhancement in Section 4. Discussion, conclusion and possible future work are presented in Section 5 .

\section{Related Works}

In this section we review the current knowledge and recent advances in both image analysis and BPM areas.

\subsection{Digital Image Analysis}

Digital images are a longstanding media in our daily life, from those images we take with our smartphones and share in social networks to those that produced by high technology devices such as X-ray machines, Microscopes, and MRI and CT imaging instruments. Digital image analysis, a highly demanded procedure in many applications, is the techniques of working on such images to produce and transfer semantic information from images.

In 2004, Lowe [6] applied low level image processing techniques to design and develop an efficient algorithm for detecting and describing interest points in a digital image. His algorithm has been introduced as Scale Invariant Feature Transform (SIFT) and it could able to extract feature points which are invariant regarding the rotation and scaling.

In 2011, Shotten et al. [7] presented a modern method to recover the $3 \mathrm{D}$ position of body joints from a single depth digital image. In 2011, Malakooti et al. [8] developed a one dimensional cellular automata based strategy for active digital image forgery detection. They extracted some statistical information from a digital image and then decrypt and embed the statistical information in the spatial domain of a particular image.

In 2012, Naji et al. [9] proposed such a vector quantization algorithm along with the clustering approach for human cell segmentation and detection in Electron Microscopy images. The detection rate was promising. In 2012, Moghaddasifar et al. [10] incorporated the Singular Value Decomposition with a cellular automata theory to color image forgery detection.

In 2014, Rohani et al. [11] combined Gaussian mixture model and discrete cosine transform for cell detection in microscopic images. The detection rate was about $91.23 \%$ which is promising in the field of microscopic segmentation.
In 2014, Pahlavan Tafti et al. [12] proposed an optimized multi-view framework for 3D microscopy vision. They combined differential evolution algorithm and epipolar geometry to restore a 3D surface structure of 2D images obtained by a Scanning Electron Microscope (SEM).

\subsection{Business Process Management and Business Process Management System}

In 2012, Weske et al. [5] developed a Business Process Management model based on the observation of a fact in which each product that a company provides to the market is the outcome of a number of performed activities. The paper suggested that Business processes are the key instrument to first organizing these activities and then improving the understanding of their interrelationships.

In 2003, Van Der Aalst et al. [3] has investigated a basic description on BPM which stands for a generic Bmp software system that is driven by explicit process designs to enact and manage operational business processes. Figure 1, is a historical perspective which he wanted to show some of the ongoing trends in Information Systems.

In 2014, Mišić et al. [13] examined the ability of open source BPM systems to improve the quality attributes of the traditional Information Systems and the work has offered promising results.

In 2014, Bardosi et al. [14] proposed a global shape and a local appearance model from specific object annotated X-ray images for metacarpal bones localization. They used such a machine learning approach such a random forest to train the system.

In 2015, Mousa et al. [15] have developed a BPM model that offers a well-organized and integrated strategy to a variety of business activities including documenting, simulating, and analyzing the main functionality of organizations. The work has also addressed some limitations and drawbacks of the latest studies in BPM.

In 2015, Bochon et al. [16] designed Cloud Business Process Management system. The work shows that a Process lifecycle has to be adopted to face with the challenges of Cloud Computing.

In 2015, Harmon et al. [17] showed that a Business Process Management could enable people to manage and improve their own businesses. There have been several approaches to change a business process, and their article explores the most important approaches which are classified into three different traditions. The oldest tradition is work simplification and quality control which is currently represented by Six Sigma and Lean. A second tradition is a management tradition was driven by teachers and consultants like Porter, Rummler and Hammer. The third tradition is driven by Information Technologists, focusing on process automation of all kinds. Each tradition has its heroes and its own vocabulary, and each emphasizes some practices over others. Further studies can be found in [18], [19], [20], [21]. 


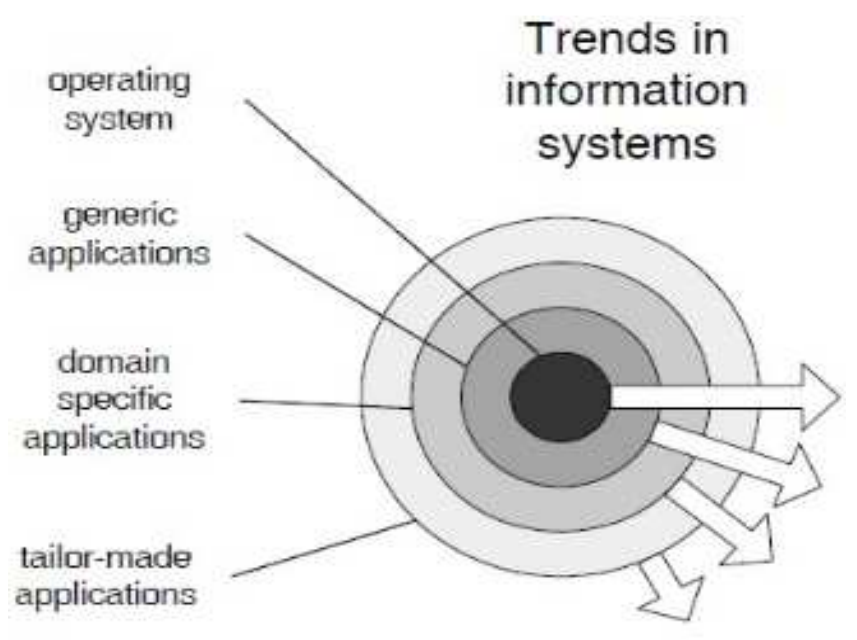

\section{From programming to} assembling.

2. From data orientation to process orientation.

3 . From design to redesign and organic growth.

Figure 1. Trends relevant for BPM.[3].

\section{Anima Image Analysis Workflow System: A Case Study}

Anima [4] which stands for ANduril IMage Analysis is a modular workflow system which enables performing image analysis in an efficient and comprehensive fashion. Anima has facilitated high throughput image analysis by providing different quality attributes including batch processing, interoperability, and platform independency, and it has been available at http://www.anduril.org/anima/.

Anima is basically targeted for analysis and algorithm developers, and it allows the combination of several computational tools to provide such a coherent workflow system. The main objective of Anima is to allow Rapid Application Development (RAD) and incorporating of new methods, without the need to port them from their original implementations. By Using Anima, the image analysis developers are able to test different methods and add newly published ones regardless of the programing languages they have been implemented in.

The main design issue of Anima is to operate as a platform to take advantage of existing software and not to exactly replace them. Anima has been ideal for developing as well as testing new algorithms, while it has been also used to run established standard analysis. It facilitates horizontal data flow schematic, which means that each step (e.g., image segmentation) could be processed with all of the images at the same process. Horizontal flow management allows making sure the processing in each step produces sensible results before performing other analyses. Since Anima is a flexible, extensible and scalable platform, it aims to simply add a new functionality into the system, and it provides exact reusability. In addition, Anima allows the measurement of attributes based on all images and the use of them later on in the analysis workflow.

\section{Applying BPM Based Attributes on Anima}

The attributes of BPM was already proven by a group of researchers in several fields. As we also discussed earlier, BPM is a cost effective and easy to use framework which aims building more efficient software application. It assists software applications of any size with designing, automating and deploying business core processes or workflows of various kinds.

One of the main reasons that the BPM has gained such widespread popularity is that it is seen as a way to realize many of the goals of having agile, structural, workflow based, and process oriented computing environments. In fact, the BPM is seen not only as a solution for making enterprise software applications more flexible, reusable, and efficient, thereby enabling them to fulfill very dynamic business needs, but also to reduce operational cost. Advantage of having BPM features are broadly lie in several quality attributes and it has been like a toolbox which easily provides the ability of creating digital forms and map out fully functioning workflows. BPM as a well-organized workflow system can also apply on existing applications and systems to enhance their entire functionalities and helps external users to communicate with them in an efficient fashion.

Table 1 addresses several BPM attributes which can apply on Anima, the case study of image analysis application which we have mentioned in Section 3. These properties would be able to make Anima more reusable, valuable, and flexible to respond to dynamic user demands in a timely manner, which could definitely impact go-to-market speed and find better business opportunity.

As you can see in Table 1 (The third row), Workflow based and process oriented attributes are key benefits of BPM, especially from the design and development perspectives. They facilitate the development and deployment of applications without the complexity and cost of need modifying underlying structures, providing complete life cycle management using a well-organized approach. Speed up the implementation is another key benefit of BPM which aims reducing the execution time of processes and enable them to improve the speed of business itself. 
Table 1. BPM properties which could potentially apply on medical image analysis.

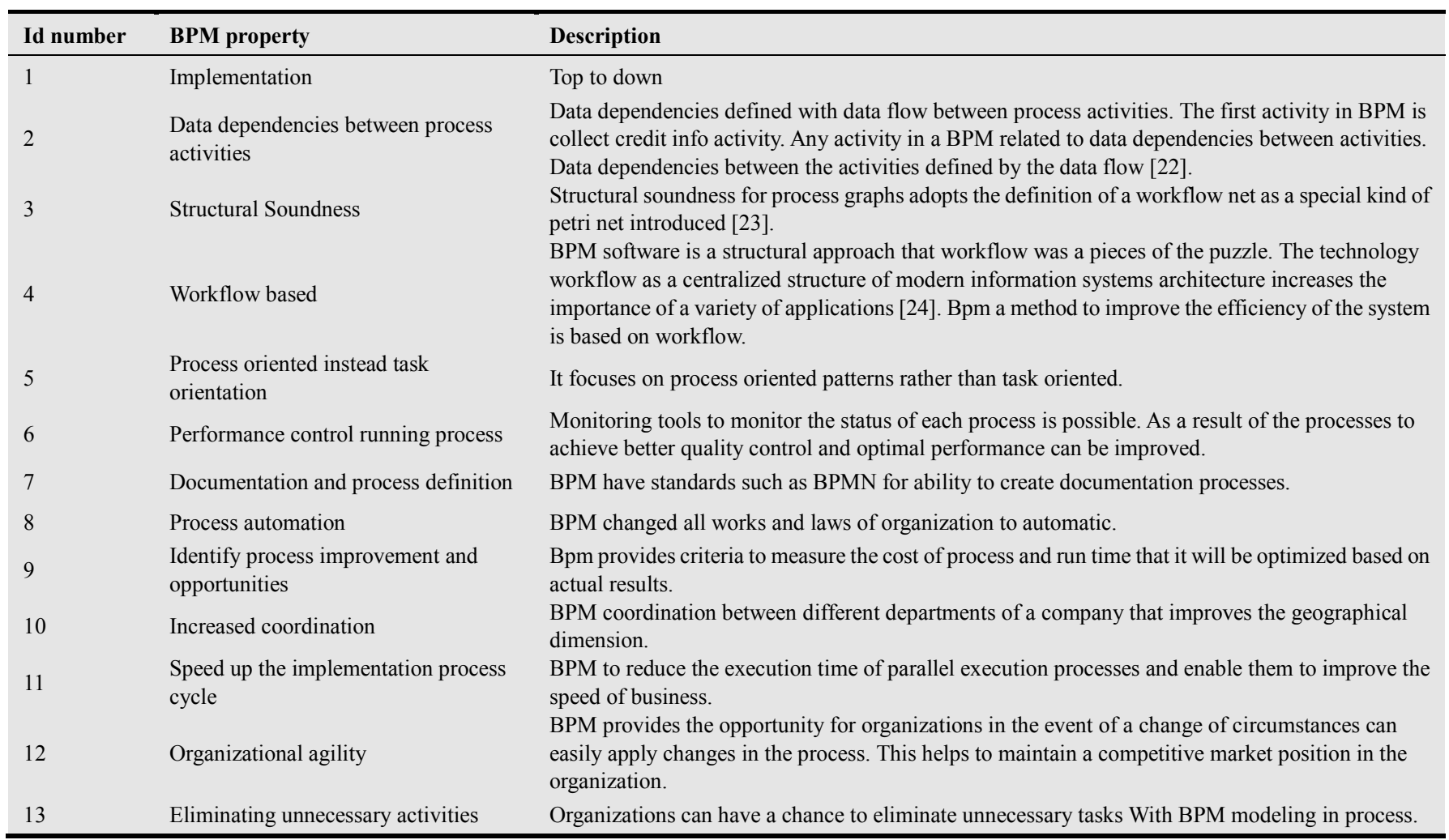

\section{Conclusion and Future Work}

In this contribution, a study is done to apply Business Process Management (BPM) as a very well-known process management system on digital image analysis applications to make them more valuable and flexible to respond to dynamic user demands in a timely fashion. After a brief introduction to BPM and digital image, several recent works on both image analysis and BPM are reviewed. We then introduce our case study, Anima, which is a modular workflow system that enables performing image analysis in an efficient and comprehensive manner. We can see that using BMP strategies will improve different quality attributes of such a digital image analysis system and will enable them to fulfill very dynamic business needs exist in the market. In this work, we pointed out a very big picture of applying BPM on image analysis tools. For future work, we will further discuss business and technical considerations and explore best practices for building and managing flexible and consistent software applications for the purpose of digital image analysis. As an additional interesting direction, we may examine the potential advantages of SaaS architectural design which newly presents in computer vision area [25] to turn medical image analysis applications into a WWW service available through the Internet.

\section{References}

[1] Gonzales, R. C. and Woods, R. E. (2008). Digital Image Processing. Prentice Hall, USA.
[2] Shapiro, L. G. and Stockman, G. C. (2011). Computer Vision. Prentice Hall, USA.

[3] Van Der Aalst, W. M. P. (2003). Challenges in business process management: Verification of business processes using Petri nets. Bulletin of the EATCS, 80, 174-199.

[4] Rantanen, V., Valori, M., \& Hautaniemi, S. (2014). Anima: modular workflow system for comprehensive image data analysis. Frontiers in bioengineering and biotechnology, 2.

[5] Weske, M. (2012). Business process management: concepts, languages, architectures. Springer Science \& Business Media.

[6] Lowe, D. G. (2004). Distinctive image features from scale-invariant keypoints. International Journal of Computer Vision, 60(2):91-110.

[7] Shotten, J., Fitzgibbon, A., Cook, M., Sharp, T., Finocchio, M, Moore, R., Kipman, A., and Blake, A. (2011). Real-time human pose recognition in parts from a single depth image. CVPR 2011.

[8] Tafti, A. P., Malakooti, M. V., Ashourian, M., and Janosepah, S. (2011). Digital image forgery detection through data embedding in spatial domain and cellular automata. In proceeding of the International Conference on Digital Content, Multimedia Technology and its Applications (IDCTA), pages 11-15.

[9] Tafti, A. P., Naji, H. R., and Malakooti, M. V. (2012). An efficient algorithm for human cell detection in electron microscope images based on cluster analysis and vector quantization techniques. In proceeding of the Second International Conference on Digital Information and Communication Technology and it's Applications (DICTAP), pages 125-129. 
[10] Tafti, A. P., Rohani, F. Malakooti, M. V., and Moghaddasifar, M A. (2012). RGB digital image forgery detection using Singular Value Decomposition and one dimensional Cellular Automata. In Proceeding of the International Conference on Computing Technology and Information Management (ICCM), pages 483-488.

[11] Rohani, F. Hassannia, H., Moghaddasifar, M. A., and Sagheb, E. (2014). Human cell detection in microscopic images through Discrete Cosine Transform and Gaussian Mixture Model. Computational Biology and Bioinformatics, 2(4): 52-56.

[12] Tafti, A. P., Kirkpatrick, A. B., Owen, H. A., and Z. Yu. (2014). $3 \mathrm{D}$ microscopy vision using multiple view geometry and differential evolutionary approaches. The $10^{\text {th }}$ International Symposium on Visual Computing (ISVC), LNCS 8888, pages 141-152.

[13] Mišić, D., Mišić, M., MilanTrifunović, T. A., \& Matić,(20124) P. AHP Based Comparison of open-source BPM Systems.

[14] Bardosi, Z., Granata, D., Lugos, G.., Tafti, A. P., Saxena, S. (2014). Metacarpal Bones Localization in X-ray Imagery Using Particle Filter Segmentation. arXiv preprint arXiv:1412.8197.

[15] Mousa, A. H., Shiratuddin, N., \& Bakar, M. S. A. (2015). Process Oriented Data Virtualization Design Model for Business Processes Evaluation (PODVDM) Research in Progress. Jurnal Teknologi, 72(4).

[16] Bochon, I., Ivens, V., \& Nagel, R. (2015). Challenges of cloud business process management. In Cloud Computing for Logistics (pp. 119-139). Springer International Publishing.
[17] Harmon, P. (2015). The scope and evolution of business process management. In Handbook on Business Process Management 1 (pp. 37-80). Springer Berlin Heidelberg.

[18] Anntonucci, I. L., \& Goeke, R. J. (2009). Analysis of Business Process Management Skills and Characteristics. Widener University, May, 7.

[19] Van der Aalst, W. M., Reijers, H. A., Weijters, A. J., van Dongen, B. F., De Medeiros, A. A., Song, M., \& Verbeek, H. M. W. (2007). Business process mining: An industrial application. Information Systems, 32(5), 713-732.

[20] Van der Aalst, W. M. (2007). Exploring the CSCW spectrum using process mining. Advanced Engineering Informatics, 21(2), 191-199.

[21] Van der Aalst, W. M. P. (2010). Challenges in business process mining. Applied Stochastic Models in Business and Industry (to appear).

[22] Malik, T. S. (2009). PROCESS MANAGEMENT: Practical Guidelines to Successful Implementation. Global India Publications.

[23] Dustdar, S., Fiadeiro, J. L., \& Sheth, A. (Eds.). (2006). Business Process Management: 4th International Conference, BPM 2006, Vienna, Austria, September 5-7, 2006, Proceedings (Vol. 4102). Springer Science \& Business Media.

[24] Zur Muehlen, M. (2004). Workflow-based process controlling: foundation, design, and application of workflow-driven process information systems (Vol. 6). Michael zur Muehlen.

[25] Tafti, A. P., Hassannia, H., Yu, Z. (2015). siftservice.com Turning a Computer Vision Algorithm into a World Wide Web Service. arXiv preprint arXiv:1504.02840. 